\title{
NUMERICAL INVESTIGATION OF THE BEHAVIOUR OF RC WIDE BEAMS UNDER IMPACT LOADS
}

\author{
Ali A. Abbas ${ }^{1}$ and Demetris M. Cotsovos ${ }^{2}$ \\ ${ }^{1}$ Senior Lecturer in Structural Engineering, School of Architecture, \\ Computing and Engineering, University of East London, London, UK \\ e-mail: abbas@uel.ac.uk \\ ${ }^{2}$ Lecturer in Structural Engineering, Institute of Infrastructure and \\ Environment, School of the Built Environment, Heriot-Watt \\ University, Edinburgh, UK
}

Keywords: Reinforced concrete, finite elements, dynamic analysis, wide beams, impact.

\begin{abstract}
The present work sets out to investigate numerically the dynamic responses of simply-supported reinforced concrete $(R C)$ beams under impact loading. The beams comprise wide sections and thus can be also considered as one-way slab panels often used in pre-cast concrete floor construction. The study was carried out using dynamic Non-linear FiniteElement Analysis (NLFEA) and was validated using published experiential data on RC wide beams tested using a drop-weight at high rate. The numerical predictions obtained show that that the response of the $R C$ wide beams under impact loading differs significantly from that established under equivalent static loading. This change predominantly takes the form of an increase in the maximum sustained load which is primarily attributed to (i) the response of a part of, rather than the whole, structural element and (ii) the development of inertia forces rather than material strain-rate sensitivity. The numerical study is based on the assumption that the effect of high loading rates on the behaviour of structural concrete is mainly linked to the development of inertia forces and not the strain-rate sensitivity of its material properties. Thus, the emphasis is on investigating the effect of loading rate on important aspects of structural response (e.g load-deflection curves, deformation profiles, load-carrying capacity, reaction forces, crack patterns and modes of failure) in an attempt to provide insight into the effect of loading-rate on the mechanics underlying $R C$ structural dynamic response. It is also important to consider that during drop-weight testing it is not easy to correlate the measured response to the actual physical state of the specimens as the maximum value of the contact force generated during impact frequently corresponds to a specimen physical state characterized by high concrete disintegration and low residual strength and stiffness. Therefore, the true load-carrying capacity is likely to be significantly lower than the maximum value of the measured applied load. As a result the validated numerical models developed are employed for conducting a parametric investigation in order to determine the true load-bearing capacity of the examined structural forms under different intensities and loading rates characterising the applied load.
\end{abstract}




\section{INTRODUCTION}

The need to address the growing demands for improving a variety of services (e.g. faster transportations, higher energy production, manufacturing and housing) has led to the construction of increasingly intricate structures. High-rise buildings, tunnels, bridges, slab-track for high-speed railways, off-shore and marine structures, storage and industrial facilities as well as nuclear power-plants are just some examples of such structures, the majority of which is fully or partially constructed from reinforced concrete (RC). Considering the high construction costs associated with such structures and their importance to the economy and society, it is essential that they achieve an elevated level of resilience and safety in order to sustain the action of loads (such as, for example, those generated by production activities, high-speed trains, accidents due to collisions or explosions, natural disasters or even acts of terrorism) induced at rates and intensities significantly higher than those of the dynamic loads considered by current design codes.

It has been established both experimentally [1-5] and numerically [6-9] that RC structural response exhibits significant departures from that observed under equivalent static (low-rate) loading once certain thresholds of applied loading rate are surpassed. Such changes become more pronounced with increasing loading rates and primarily take the form of an increase in the maximum sustained load and stiffness. Impact loading is a characteristic example of highrate loading and is generated during the collision of an object onto a certain area of a structure. Such loads are applied locally and their form (i.e. intensity, duration, loading-rate and timehistory) depends on the mass, velocity and shape of the object, the dynamic characteristics of the structural element (i.e. mass and stiffness) and the properties of the contact area. The application of such loads on RC structures results in the generation of stress waves, the propagation of which, within a heterogeneous material such as concrete results in the development of a complex triaxial stress field [10-12]. This field is further accentuated by the cracking processes of concrete and the deflection of the waves on the developing cracks and on the boundaries of the structure. Hence, defining the response of RC structures under high rate loading is a complex wave-propagation problem within a highly nonlinear medium. The available numerical and test data reveals that, with increasing loading rates, RC structural response such as deflection and cracking profiles becomes more localised. This is because the portion of the $\mathrm{RC}$ element reacting to the external load reduces in length as failure occurs prior to the generated waves reaching the supports [7,8]. This phenomenon, combined with the inertia forces developing along the element span in the transverse directions, underlie the mechanisms governing RC structural responses [7,8]. The observed change in these responses is attributed by many researchers to the strain-rate sensitivity that is assumed to characterize the material properties of concrete and steel. Many constitutive models used to describe the behaviour of concrete subjected to high loading rates assume that the concrete material properties are dependent on strain-rate. In contrast with this view, recent work investigating the fundamental behaviour of concrete by modelling small cylinder specimens $[10,11]$ found that concrete material properties are essentially independent of stain rate and that the effect of loading rate on the specimen behaviour is primarily attributed to the inertia forces which develop within a specimen and the effect is at the structural rather than the material level.

The strain-rate independent assumption has been also employed in the present work in order to ascertain the significance of the role that inertia plays in the beam's dynamic response when subjected to high loading rates. Thus, the presently-examined wide RC wide beam specimens were modelled by means of static material properties of concrete coupled with the dynamic characteristics of the system at a structural level. The numerical study uses published experimental data on wide RC beams [1] and aims at studying the mechanics underlying $\mathrm{RC}$ 
structural response under high rate loading conditions through the use of a commercial nonlinear finite-element (NLFEA) analysis package ANSYS [13]. After validating the finiteelement (FE) predictions obtained against their experimental counterparts, emphasis was focused on investigating the effect of loading rate on important aspects of RC structural responses such as load-deflection curves, deformation profiles, load-carrying capacity, reactions, crack patterns and modes of failure. During drop-weight testing it is difficult to correlate the measured responses to the actual physical state of the specimens as the measured maximum value of imposed load frequently corresponds to a specimen that is already disintegrated and exhibiting low residual load-bearing capacity and stiffness. This stage of structural response has little practical significance as it depends heavily on post-failure mechanisms for transferring the applied loads to the specimen supports. Therefore, the true load-carrying capacity is likely to be significantly lower than the maximum value of measured applied load. To address this, a parametric study was carried out to determine the true load-bearing capacity under different intensities and loading-rates of the imposed impact load.

\section{EXPERIMENTAL BACKGROUND}

A large number of experiments has been carried out to date to investigate the responses of RC beams under impact loading [1-5]. In the majority of these cases, the load is applied by means of a steel mass (impactor) allowed to fall onto the mid-span region of the specimen from a predefined height depending on the desired rate of loading. The ratio of the mass of the steel impactor to the mass of the RC beam specimen used in the various case studies vary significantly, ranging from low values of $17 \%$ (the steel impactor being much lighter than the RC specimen) to large values such as $150 \%$ (the steel impactor being heavier than the RC specimen). The load is usually applied through the use of pads or platens (made of steel, ply or rubber) in order to moderate the damage in the area of contact between the steel impactor and the RC specimen. The duration of loads generated during drop-weight tests is usually extremely short (i.e. a few milliseconds) and the intensity of the applied load increases rapidly from zero to a maximum value. Typically, the variation of strain and displacement at various points along the specimen length, the velocity and acceleration of the steel impactor, as well as measurements of the support reactions are recorded. In addition, crack formation and propagation up to failure, is closely monitored as it provides an indication of the corresponding internal stress state of the beam at every stage of the loading process. However, it should be noted, that the latter information is not usually available in literature. Information concerning crack patterns and deformation profiles is usually recorded after (and not throughout) the application of the impact load. Therefore, as explained earlier, this usually relates to a specimen of little practical use as it is heavily degraded and is dependent on post-failure mechanisms for transferring the applied loads to the supports. This is often achieved through the use of a high-speed video camera. Similar RC beams to those tested under impact loading are usually also tested under static loading, for purposes of comparison.

Typical load deflection curves are shown in Figure1a describing the responses of RC beams under impact and equivalent static load exerted at mid-span, which reveal that an increase in loading-rate leads to an increase in the maximum sustained load and to a stiffer response. The corresponding deflected shapes indicate that, as the rate of the applied load increases, the portion of the RC beam mostly affected by the application of the external load tends to concentrate at the mid-span region of the specimen, where the load is exerted and as a result cracking tends to concentrate in a region of the $\mathrm{RC}$ beam around the point at which the external load is applied. This localised response increases with increasing loading rates. The variation in the recorded peak value of the applied load, i.e. contact force generated dur- 
ing impact, (maxPd) expressed as a ratio of its maximum static counterpart (maxPs) is shown in Figure 1b. The data indicates that an increase in the rate of applied loading results in the $\mathrm{RC}$ beam being able to sustain higher levels of loading. Data obtained from such tests is characterised by a large scatter partly due to a wide range of parameters (associated with the experimental setup and the specimen) which differ from test to test. This scatter predominantly reflects the difficulty in correlating the measured responses under impact load to the actual physical state of the specimens discussed earlier. This is different to the way the load carrying capacity term is usually defined and understood under static loading. So in design terms, one has to be careful in estimating the maximum impact load that can be sustained by a given beam as this will depend on the level of damage that can be tolerated and the residual strength required following impact.
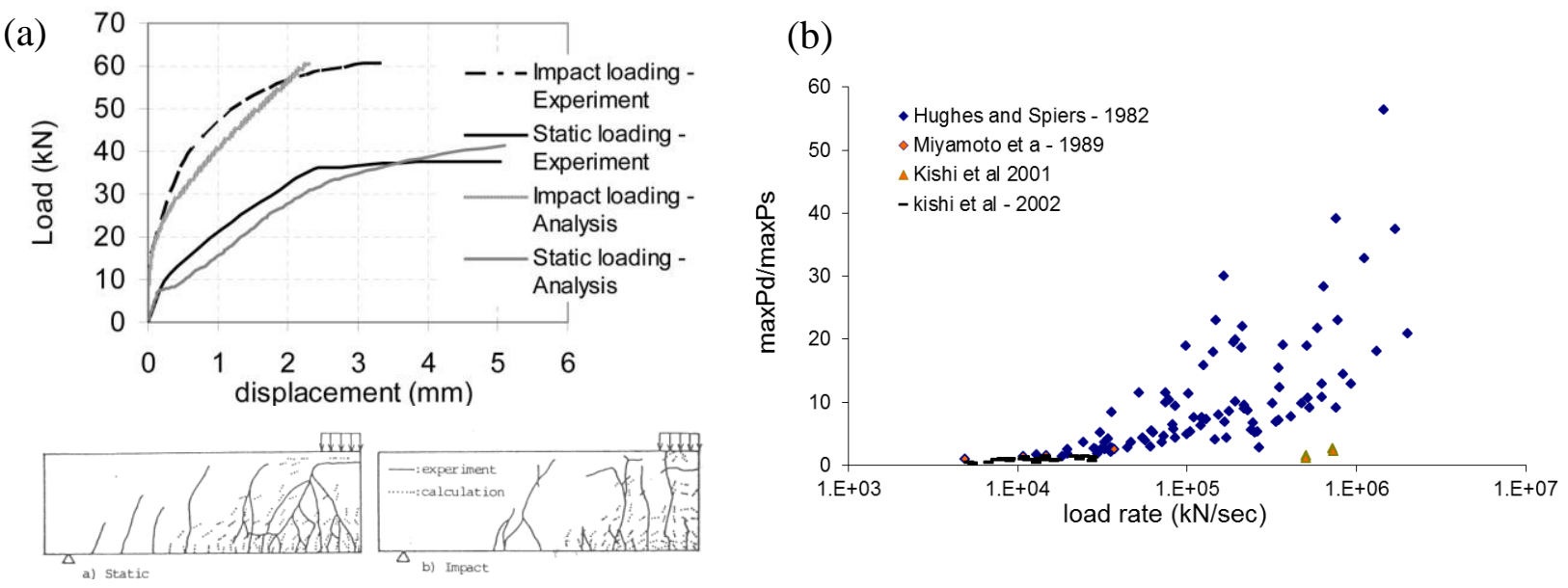

Figure 1: Experimental background on (a) static and impact tests [9] along with numerical counterparts and (b) scatter in impact test data

\subsection{Specimens considered}

Published experimental data on RC wide beams [1] forms the basis of the present numerical study and the salient features of the specimens are depicted in Figure 2. Each specimen was $1800 \mathrm{~mm}$ in length, $360 \mathrm{~mm}$ in width and $125 \mathrm{~mm}$ in thickness and was simply supported on rollers near the ends of its span in the longitudinal direction, forming a clear span of 1600 $\mathrm{mm}$. The static responses were initially established under monotonic loading applied until failure and then under impact loading via drop-weight testing, which was achieved by dropping a $38 \mathrm{~kg}$ steel impactor with a flat contact face from a height of $5 \mathrm{~m}$ (thus achieving a velocity of $10 \mathrm{~m} / \mathrm{sec}$ on impact). The ratio of the mass of the steel impactor to that of the beam is approximately $13 \%$ which is towards the lower end of corresponding values in the majority of experimental investigations. This is considered advantageous to using heavy drop weights which usually result in a complete disintegration of the test specimen thus making it more difficult to derive meaningful conclusions regarding the specimens' response. Both static and impact loads were applied through a steel cross-beam placed on top of the RC beam extending along the full width of the RC beam, in order to better distribute the applied load on the concrete surface and avoid the development of local stress consecrations which can in turn cause cracking in the region of the beam were the external load is exerted. The mean cube compressive strength of the concrete was found to be $64.30 \mathrm{MPa}$, while the mean tensile splitting strength was found to be $3.94 \mathrm{MPa}$. The mean ultimate strength and strain was found to be $670 \mathrm{MPa}$ and 0.067 , respectfully. 

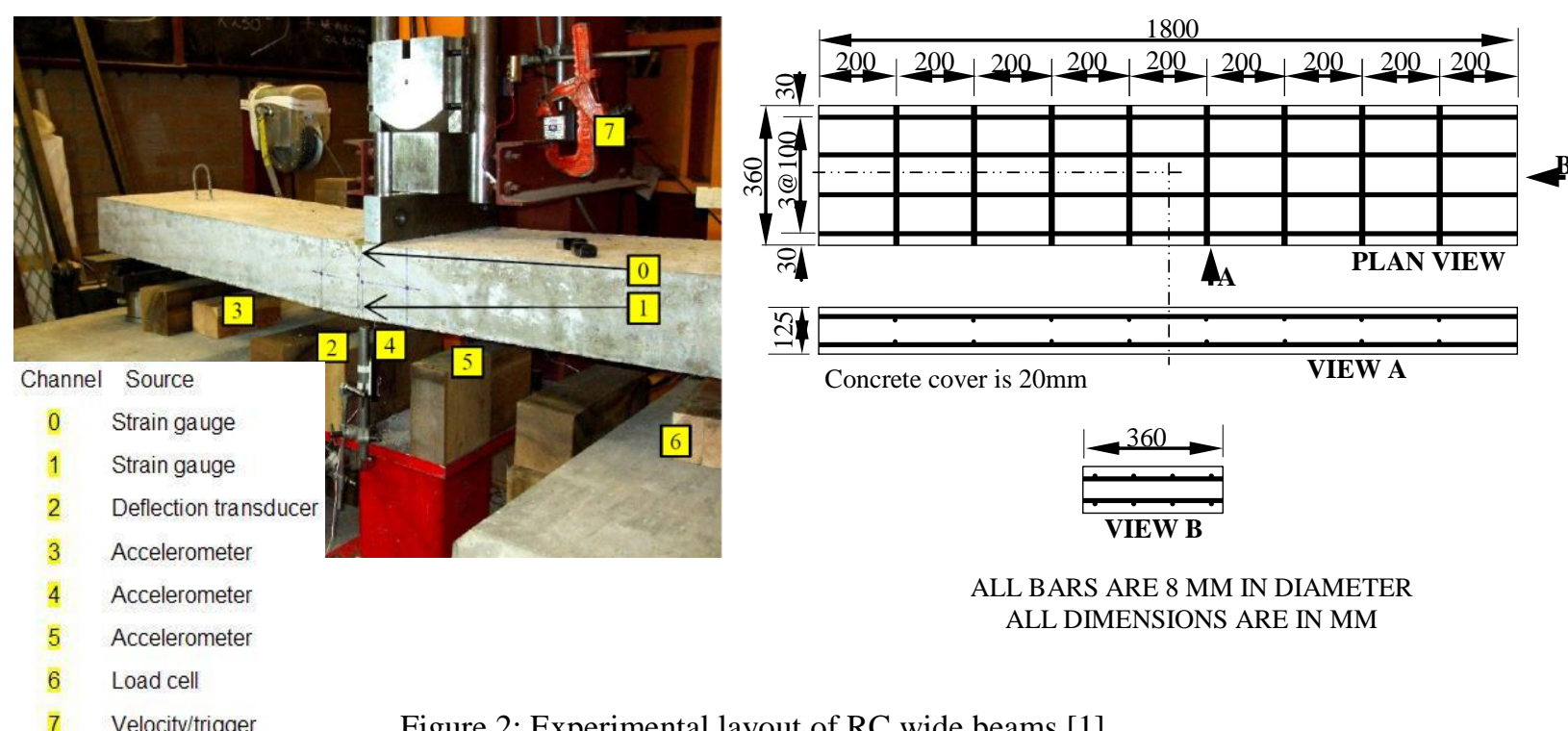

ALL BARS ARE 8 MM IN DIAMETER ALL DIMENSIONS ARE IN MM

$\begin{array}{lll}7 & \text { Velocity/trigger }\end{array}$

Figure 2: Experimental layout of RC wide beams [1]

\subsection{Response under static loading}

The load-deflection curves describing the response of the $\mathrm{RC}$ beam under static load is presented in Figure 3. The specimen initially behaved elastically up to around $12 \mathrm{kN}$ and cracking started forming at mid-span at the lower face of the beam. As the loading was increased, cracking extended upwards resulting in a gradual reduction in the stiffness of the beam. The maximum load carrying capacity was found to be $32 \mathrm{kN}$ beyond which the load decreased gradually followed by

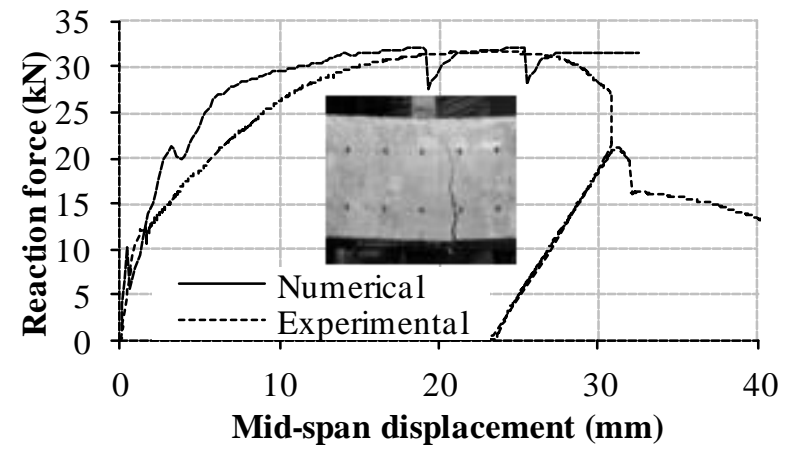

Figure 3: Experimental [1] and numerical results under static loading an abrupt reduction in the load carrying capacity due to failure in the compressive zone of the beam at mid-span. The FE-based load-deflection curve is also depicted on Figure 3 which shows good agreement with corresponding experimental data.

\subsection{Response under impact loading}

Figure 4a shows the load generated during impact whereas the load deflection curves in Figure $4 \mathrm{~b}$ indicates that under high-rate loading conditions, the response of the $\mathrm{RC}$ wide beam becomes stiffer while allowing the beam to attain higher levels of loading. Furthermore, under impact loading cracking initiates at a much earlier stage in the loading process compared to that observed under static loading while at the same time these cracks propagate much faster extending rapidly towards the upper face of the beam. The photographic record confirms that cracking initiates at the lower surface of the mid-span region of the beam and almost immediately extends upwards. The crack pattern indicates that the beam exhibits a localised response confined to its middle portion during the first stages of the experiment. The crack has a vertical flexural form that penetrates deeply into the compressive zone. This is a typical mode of failure which corresponds to the specimen's true load-carrying capacity (i.e. relating to the specimen still acting as a continuum). This appears to occur within a very short period of time 
that is much less than the duration of the whole test, beyond which the experimentally established behaviour is likely to describe post-failure phenomena of little practical significance.
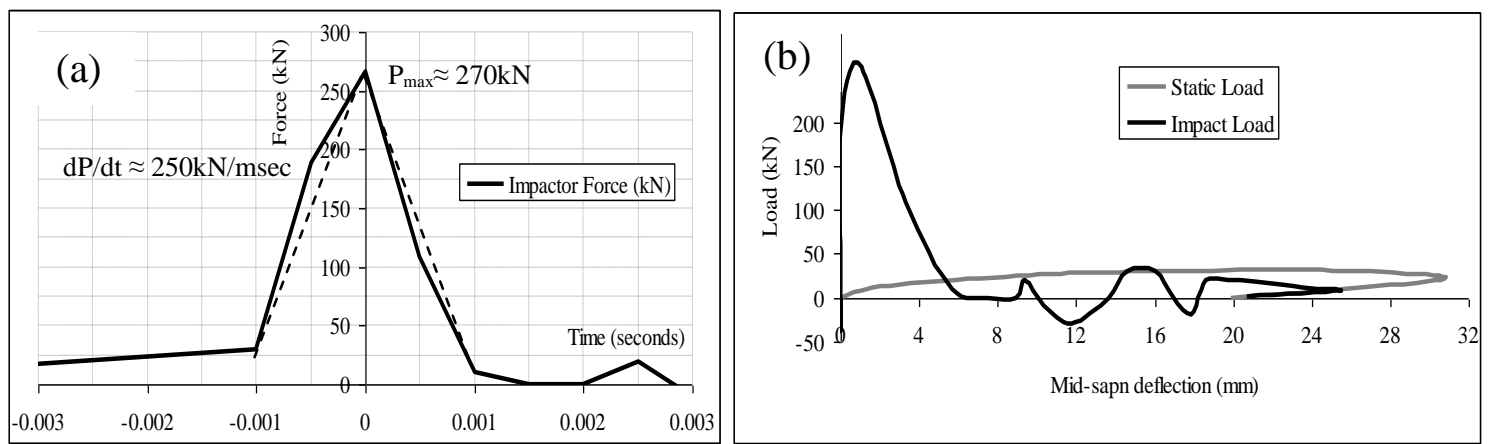

Figure 4: Experimental data [1] showing (a) impact load history and (b) static and impact load-deflection curves

\section{FE MODELING STRATEGY}

Due to the difficulties and limitations associated with RC element tests under high-rate loading, resort has been made to the use of nonlinear FE analysis. Use of the FE method can provide a more detailed description of the structural response and also allows the investigation to be extended to structural forms more complex than the simple RC structural elements that can be studied experimentally. Another advantage of the numerical investigation is that, unlike testing, which relies on measuring and recording the effect of the rate of loading on structural behaviour, it allows a more comprehensive study of the causes of change in the behaviour exhibited by the RC structural elements. In order to accurately model the problem at hand, it is necessary to fully identify the complexities that characterise it. The FE analysis code adopted for the current investigation (ANSYS) is capable of carrying out 3D non-linear static and dynamic analyses, employing appropriate material models capable of accounting for the non-linear behaviours of concrete and steel. Due to the non-linear behaviour of concrete, the governing equation of motion is solved numerically through the implicit Newmark integration scheme $[8,14]$. Provided that the stability criteria are observed, the method yields comparable results with explicit FE formulations in RC element analysis. A brief description of the model parameters is provided herein for completeness.

\subsection{Concrete material modelling}

ANSYS is a well-known commercial FEA package that is widely used in a variety of complex structural problems. The present study uses a concrete model derived based on regression analysis of test data on concrete cylinders and cubes subjected to uniaxial compression and tension under quasi-static load rates. The ensuing stress-strain curves are depicted in Figures $5 \mathrm{a}$ and $5 \mathrm{~b}$, respectively.

(a)

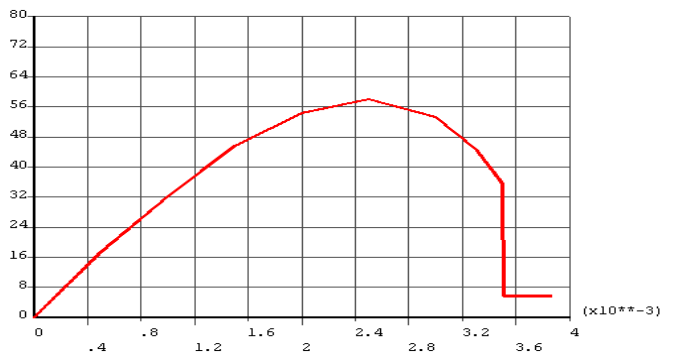

(b)

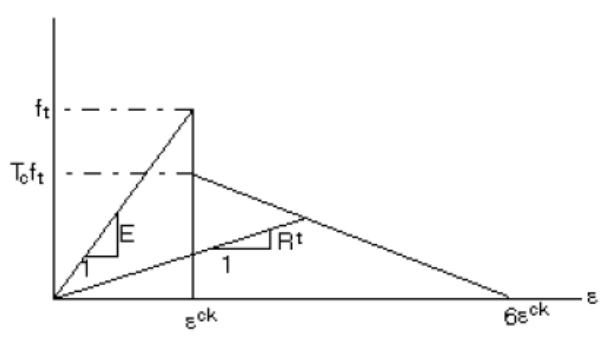

Figure 5: Uniaxial stress-strain relations adopted in ANSYS for concrete under (a) compression and (b) tension 
By assuming that concrete is an orthotropic material, the uniaxial stress-strain curves described above are applied to each principal-stress axis, thus leading to a formulation of a general constitutive model describing the behaviour of concrete under triaxial loading conditions. The analytical formulation in ANSYS also defines the slope of the descending softening branch of the tensile stress-strain curve. The default value is 0.6 , the effect of the variation of this parameter was not investigated in the present study.

\subsection{Reinforcement modelling}

Steel reinforcement is explicitly included in the FE model using one-dimensional bars under uniaxial tension and compression only. Full bond is assumed between steel and concrete, with local bond transfer bounded by the tensile capacity of the concrete at the Gauss points near the reinforcement. Steel constitutive behaviour follows a simple bilinear hardening model accounting for the initial elastic and an averaged post-yield behaviour of the bars (see Figure 6). Similarly to the concrete case, the effect of the loading-rate on steel behaviour is discounted at the material level.

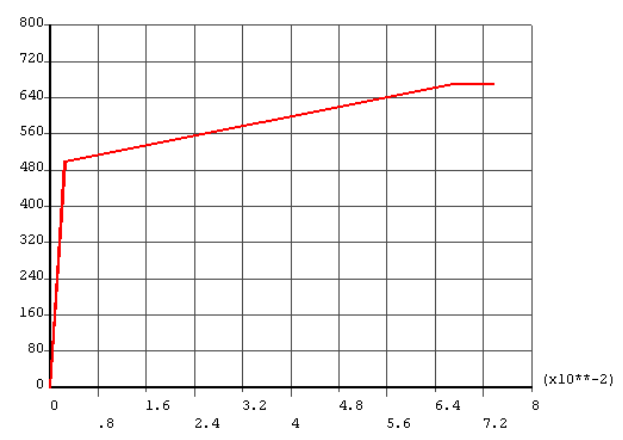

Figure 6: Uniaxial stress-strain relations for steel bars

\subsection{Crack modelling}

Following the modelling capabilities of the adopted FE analysis program (ANSYS), cracking is modelled using the smeared-cracking approach. Following this modelling convention, cracks are allowed to form at the integration points where stress evaluation takes place and their effect is spread within a region associated with a particular Gauss point.

\subsection{FE mesh and loading}

The concrete medium is modelled by using a dense mesh of 8-noded brick elements with an edge size of between $20 \mathrm{~mm}$ to $30 \mathrm{~mm}$ and the FE mesh is depicted in Figure 7. The element formulation adopts a reduced integration scheme to avoid numerical problems due to locking. Reinforcement bars are modelled by 2-noded single Gauss point truss elements with cross-sectional areas distributed to the relevant nodes of the beam's cross-section so as to be equivalent, in terms of both cross-sectional area and location, to the actual reinforcement of the beams. Because of the double symmetry of the problem at hand, a $100 \mathrm{~mm}$

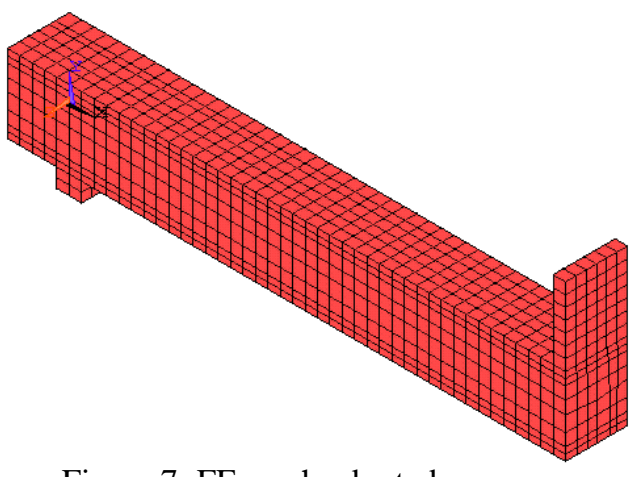

Figure 7: FE mesh adopted strip of the concrete slab was modelled with suitable boundary conditions. As in the case of the experimental investigation, the external load is applied at mid-span of the RC beam through a rigid element. The value of the applied load increases linearly at a constant rate until the load-carrying capacity of the RC beam is reached and failure occurs. Initially the static problem is investigated in order to effectively calibrate the model followed by the investigation of the dynamic problem. Various rates of loading are investigated herein ranging from $250 \mathrm{kN} / \mathrm{sec}$ to $250,000 \mathrm{kN} / \mathrm{sec}$. the latter being equivalent to the loading rate applied during the drop-weight discussed earlier. 


\section{PREDICTED FE RESPONESES UNDER STATIC LOADING}

In the FE modelling of the static test, the external load is applied in the form of displacement increments at mid-span and the load-deflection results obtained are presented in Figure 3 which, as pointed out earlier, compares well with experimental data. Overall, the numerical investigation predicts a response similar to that established experimentally, yielding very similar values of load-carrying capacity and maximum deflection $(30 \mathrm{kN}$ and $30 \mathrm{~mm}$, respectively). Figure 8a shows the deformed shape of the beam in the case of static loading, whilst the cracking pattern presented in Figure 8b, which shows that flexural cracks begin to appear in the mid-span region of the specimen and gradually extend towards the supports as the load increases. The cracking pattern correlates closely with its experimental counterpart.

(a)

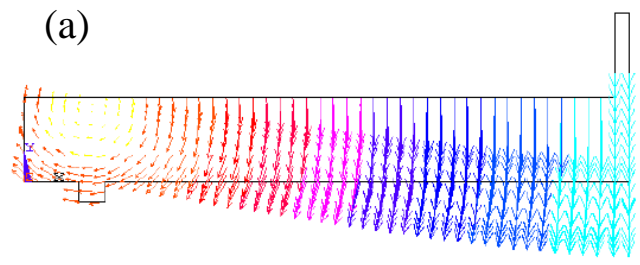

(b)

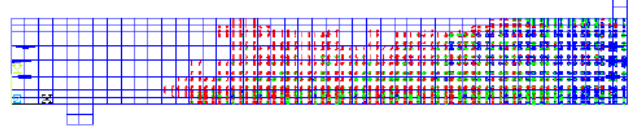

Figure 8: Static-load FE results showing (a) deflected shape and (b) cracking pattern

\section{PREDICTED FE RESPONESES UNDER HIGH RATE LOADING}

In the dynamic case studies, the effect of a number of different rates of loading on the dynamic responses of the $\mathrm{RC}$ wide beam was investigated numerically. The loading rates considered were $2.5,25$ and $250 \mathrm{nkN} / \mathrm{msec}$ (the latter being equal to the loading rate applied during the drop-weight test [1] discussed earlier). The load is applied monotonically up to failure at a constant rate in order to study the dynamic responses of the subject specimens. The numerical predictions obtained are presented in Figure 9a in the form of applied load versus mid-span deflection curves, which reveal a considerable increase in stiffness and maximum sustained load with higher values of applied loading rate and at the same time, a decrease in the maximum deflection at mid-span. However, it is also evident that the level of loading applied during testing (approximately $270 \mathrm{kN}$ at a rate of $250 \mathrm{kN} / \mathrm{msec}$ ) is considerably lower than the maximum value of imposed load obtained numerically for the case of monotonic loading applied with the same loading rate. Nevertheless, the numerically predicted value of the mid-span deflection at $270 \mathrm{kN}$ is similar to that established experimentally thus providing evidence concerning the validity of the numerical model presently employed.

The deformation profiles prior to failure, shown in Figure9b, suggest that as the rate of loading increases the response of the beam tends to become more localised resulting in the reduction of the portion of the element affected by the application of the external load which tends to concentrate at the mid-span region of the beam. In particular, as the rate of loading increases, the deflected shape of the beams progressively attains a narrowing bell-shaped form in the vicinity of the loading point (with its convex portions near the supports gradually increasing at the expense of the middle concave portion, which deflection becomes disproportionally large). The predicted crack patterns exhibited under different rates of loading prior to failure (see Figure 9c) are consistent with the deformed profiles of the beam, indicating that as the rate of loading increases, cracking becomes more localized primarily around the point at which the load is exerted.

Strain rates exhibited in the region adjacent to the point of application of the external load were calculated for the maximum loading rate presently considered as depicted in Figure 10. 
The strain rate is found to reach values in the range of $\pm 10 \mathrm{sec}^{-1}$ for the larger part of the duration of the loading process and only towards the final part do they become greater than 200 $\mathrm{sec}^{-1}$. Considering available tests data describing the effect of increasing loading rates on the behaviour of plain concrete under high rates of uniaxial compressive and tensile loading [1012] - see Figure 11 - it is shown that the loading rate corresponding to the above range of values of calculated strain rates (i.e. \pm 10 sec-1) may, at best, result only in a small increase in concrete strength. Even during the latter stages of the loading process the crack patterns presented earlier show that the specimen has suffered considerable cracking and therefore the calculated strain rates do not correspond to the beam acting as a continuum but to one that has disintegrated considerably.
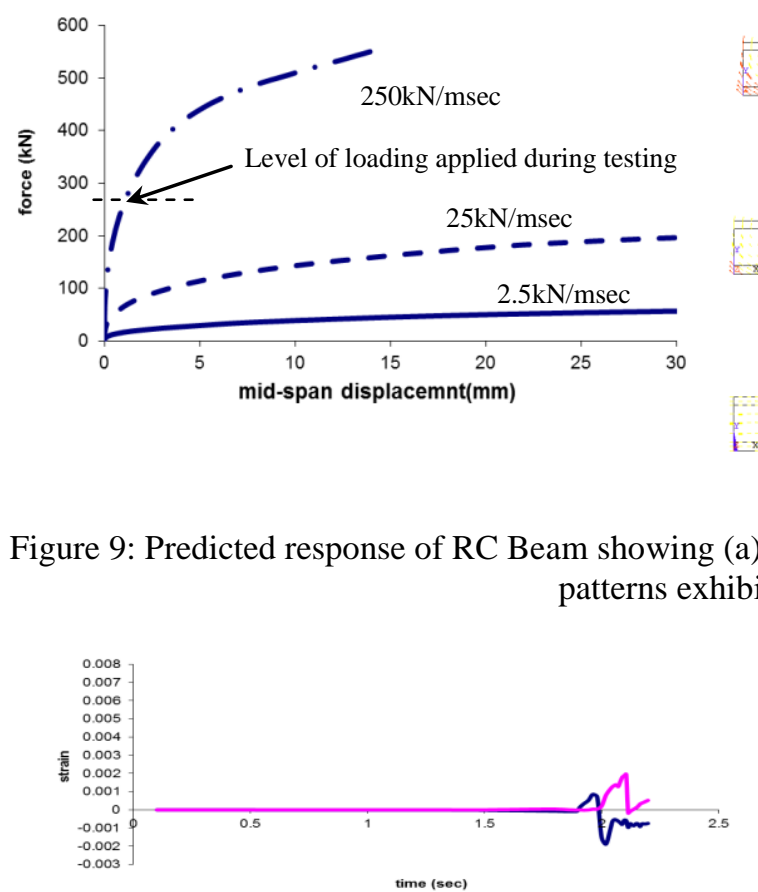

(a)

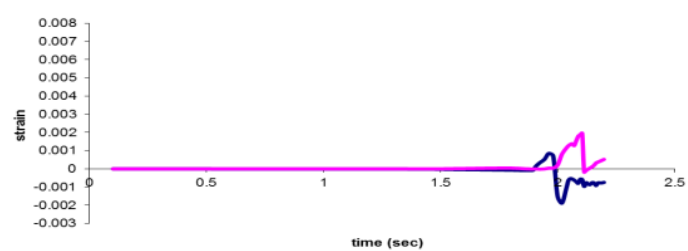

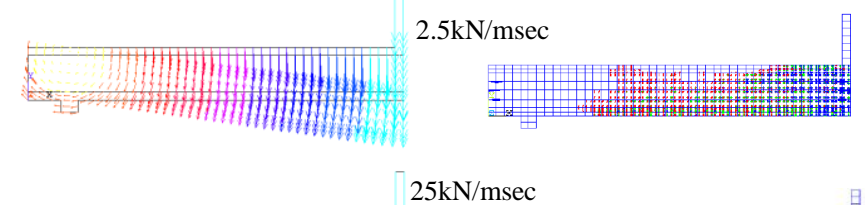

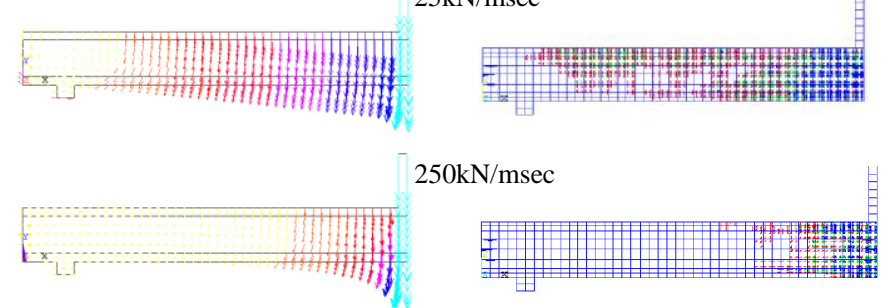

Figure 9: Predicted response of RC Beam showing (a) load-deflection curves, (b) deflected shapes and (c) crack patterns exhibited prior to failure

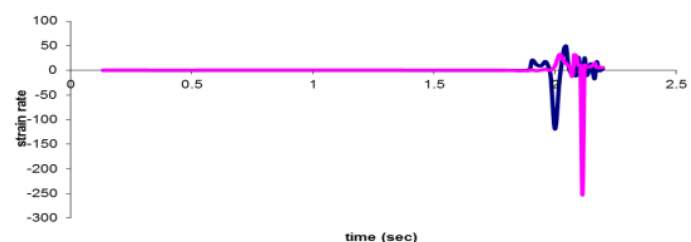

(b)

Figure 10: Predicted values of (a) strains and (b) strain rate in the region adjacent to the point of application of the external load
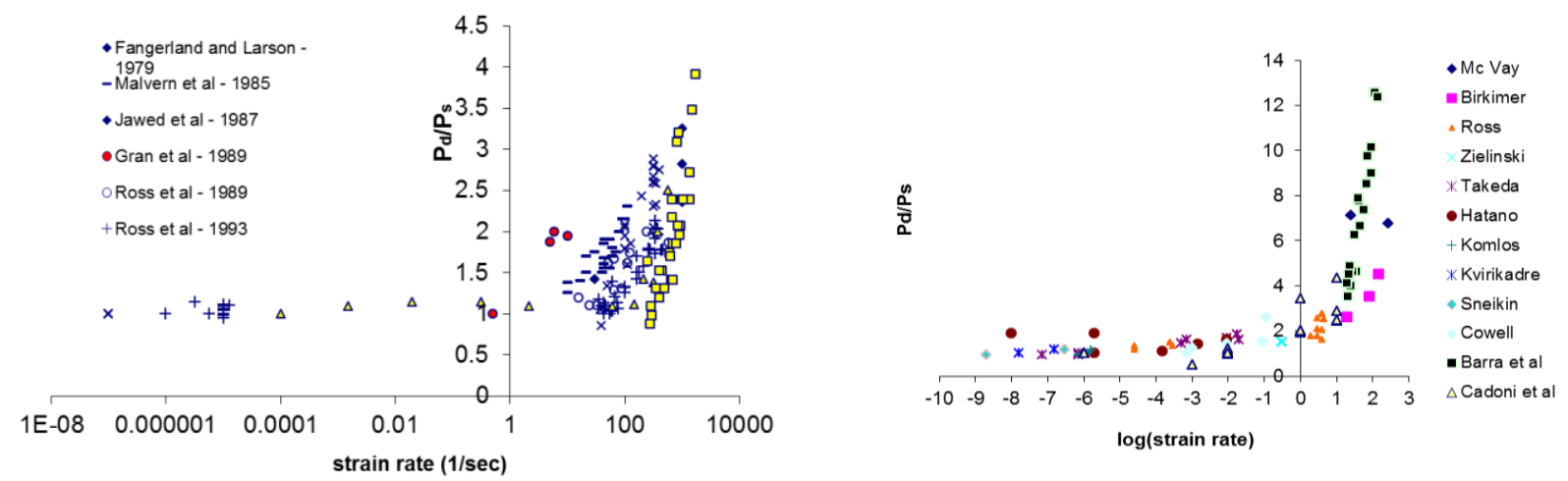

Figure 11: The effect of increasing loading rates on the behaviour of plain concrete specimens under high rates of uniaxial (a) compressive [10,11] and (b) tensile loading [12] 


\section{CONCLUSIONS}

Comparing the numerical predictions with their experimentally established counterparts describing the responses of the RC wide beams (or one-way slabs) under static and impact loading, it can be concluded that both sets of data show good agreement. In particular, both experimental and numerical investigations clearly demonstrate that during high-rate loading (i.e. impact) the specimen is capable of undertaking higher levels of loading. Nevertheless it is clearly demonstrated that the experimental investigation is unable to correlate the measured responses to the actual physical state of the specimens as the measured maximum value of imposed load corresponds to a specimen well below its load-bearing capacity established experimentally.

A comparison of the load-deflection curves under static and impact loads shows that under high-rate loading conditions, the response of the $\mathrm{RC}$ beam becomes stiffer and allows the beam to attain higher levels of loading. This change in structural response is essentially linked to the deformation profile exhibit by the beams with increasing loading rates which becomes more localised and confined to the mid-span loading region (i.e. the area at which the load is exerted) as the loading rate increases. Nevertheless the mode of failure appears to remain unaffected by loading rate.

Form the preceding analysis, it can be concluded that the numerical investigation of RC wide beams under high-rate loading yield similar results to that established experimentally using drop-weight testing. In particular, it can be suggested that the agreement observed between numerical and experimental data validates the initial assumption that the effect of loading rates on the specimen behaviour can be attributed - at least for the rates considered - to the inertia effect of the RC beam mass (which results in a reduction in the length of the beam which responds to the applied load) and not to the loading-rate sensitivity of the material properties of concrete and steel. However, more research is required in order to correlate the measured responses to the actual physical state of the specimens considered in order to accurately determine certain important aspects of RC structural response (i.e. load-carrying capacity) under increasing rates of impact load. Such information is essential in order to develop advanced analysis methodology which in turn can facilitate the development of efficient design solutions (in terms of both safety and economy) capable of safeguarding the structural integrity, resilience and performance requirements. To this end, further experimental and numerical studies are currently being conducted by the authors on a variety of structural configuration subjected to various types of high-rate loading.

\section{REFERENCES}

[1] A.A. Abbas, A.D. Pullen, D.M. Cotsovos, Structural response of RC wide beams under low-rate and impact loading. Magazine of Concrete Research, 62, 723-740, 2010.

[2] Y. Chen, I. May, Reinforced Concrete members under drop-weight impact. ICE Proceedings, Structures \& Buildings, 162, 1, 45-56, 2009.

[3] G. Hughes and D. Spiers, An investigation on the beam impact problem. Cement and Concrete Association, Technical Report 546, 1982.

[4] N. Kishi, H. Mikami, K.G. Matsuoka, T. Ando, Impact behaviour of shear- failure-type $\mathrm{RC}$ beams without shear rebar. International Journal of Impact Engineering, 27, 955968, 2002. 
[5] S. Saatci, F.Vecchio, Effects of shear mechanisms on impact behavior of reinforced concrete beams. ACI Structural Journal, 106, 1, 78-86, 2009.

[6] A.Q. Bhutti, N. Kishi, H. Mikani, T.Ando, Elasto-plastic impact analysis of shearfailure-type RC beams with shear rebars'. Materials \& Design, 30, 502-10, 2009.

[7] D.M. Cotsovos, M.N. Pavlovic, Modelling of RC Beams under Impact Loading. ICE Proceedings, Structures \& Buildings, 165, 2, 77-94,2012.

[8] D.M. Cotsovos, N.D. Stathopoulos, C. Zeris, Fundamental behaviour of RC beams subjected to high rates of concentrated loading. ASCE Journal of Structural Engineering, 134, 1839-1851, 2008.

[9] A. Miyamoto, M. King, M. Fujii, Non-linear dynamic analysis and design concepts for RC beams under impulsive loads. Bulletin of the New Zealand National Society for Earthquake Engineering, 22, 98-111, 1989.

[10] D.M. Cotsovos, M.N. Pavlovic, Numerical investigation of concrete subjected to compressive impact loading. Part 1: A fundamental explanation for the apparent strength gain at high loading rates. Computers \& Structures, 86,145-163, 2008.

[11] D.M. Cotsovos, M.N. Pavlovic, Numerical investigation of concrete subjected to compressive impact loading. Part 2: Parametric investigation of factors affecting behaviour at high loading rates. Computers \& Structures, 86,164-180, 2008.

[12] D.M. Cotsovos and M.N. Pavlovic, Numerical investigation of concrete subjected to high rates of uniaxial tensile loading. International Journal of Impact Engineering, 35, 319-335, 2008.

[13] ANSYS, Version 13.0, Swanson Analysis Systems Inc., Houston, PA, 2004.

[14] T. Belytschko, A survey of numerical methods and computer programs for dynamic structural analysis. Nuclear Engineering and Design, 37, 23-34, 1976. 